\title{
High expression of the long non-coding RNA HEIRCC promotes Renal Cell Carcinoma metastasis by inducing epithelial- mesenchymal transition
}

\author{
Jing Xiong ${ }^{1,2}$, Ying Liu ${ }^{3}$, Shengjun Luo ${ }^{1}$, Li Jiang ${ }^{1}$, Yang Zeng ${ }^{1}$, Zhixiong Chen ${ }^{1}$, \\ Xiaobo Shi ${ }^{1}$, Bufan Lv' ${ }^{1}$, Wei Tang ${ }^{1}$ \\ ${ }^{1}$ Department of Urology, The First Affiliated Hospital of Chongqing Medical University, Chongqing \\ ${ }^{2}$ Departmentof Urology and Andrology, Taihe Hospital, Hubei University of Medicine, Shiyan, Hubei Province, China \\ ${ }^{3}$ Department of Preventive Medicine, School of Public Health and Management, Hubei University of Medicine, Shiyan, Hubei \\ Province, China
}

Correspondence to: Wei Tang, email: tangwei2060@163.com

Keywords: long non-coding RNAs, HEIRCC, renal cell carcinoma, prognosis, EMT

Received: June 30, 2016

Accepted: November 04, 2016

Published: December 24, 2016

\section{ABSTRACT}

Increasing evidence indicates that long non-coding RNAs (IncRNAs) have been associated with cancer development. However, the contributions of IncRNAs to renal cell carcinoma (RCC) remain poorly characterized. Here, we identified a novel IncRNA, termed HEIRCC, which was up-regulated in RCC tissues through IncRNA microarray analysis and subsequent validation in 60 RCC clinical specimens and cell lines. The high expression of HEIRCC is associated closely with the clinical pathology features such as larger tumor size, poor differentiation, lymphatic metastasis. In vitro assays revealed that HEIRCC knockdown could inhibit cell proliferation, trigger late apoptosis, suppress cell migration and invasion. We further demonstrated that depletion of HEIRCC reduce the epithelial to mesenchymal transition (EMT) program by regulating expression levels of EMT-associated markers in RCC cells. Thus, HEIRCC might be act as an important regulator of EMT in RCC progression and might be a novel therapeutic target for the advanced RCC therapy.

\section{INTRODUCTION}

Renal cell carcinoma (RCC) counts among the major cancers that endanger human health in the world, with about 63,920 newly detected cases yearly [1]. Renal cell carcinoma incidence has increased for over two decades, and patients with advanced RCC (stage IV) have a significantly reduced five-year survival rate (less than $30 \%)$ [2, 3]. Despite the identification of several genes related to cancer development and progression malignant events still need to be characterized at the molecular level. Indeed, uncovering the exact molecular mechanisms by which renal cell carcinoma develops and progresses is essential, in order to identify novel therapeutic targets to combat RCC. Epithelialmesenchymal transition (EMT) was initially reported for its crucial role in metazoan embryogenesis as well as organ development. Emerging evidence has shown that cancer progression and metastasis are tightly associated with EMT $[4,5]$.
Long non-coding RNAs (lncRNAs), a subclass of noncoding RNAs (ncRNAs), have sequence lengths of $200 \mathrm{bp}$ and above [6, 7]. At the beginning, lncRNA molecules were merely described as cloning artifacts. However, it has become increasingly apparent that lncRNAs are involved in multiple cellular biological processes, ranging from transcriptional and posttranscriptional regulation to cell cycle distribution, cell differentiation and epigenetic modifications [8]. Interestingly, lncRNAs attract increasing attention for the elucidation of interconnected pathways regulating tumorigenesis and metastasis in multiple malignancies, including glioma, lung, colorectal and hepatocellular cancers $[9,10]$. Meanwhile, reports assessing lncRNA involvement in RCC are scarce, and the pathways regulating RCC aggressiveness remain poorly understood.

This study aimed to assess lncRNA involvement in the control of EMT as well as metastasis in RCC. The differences between IncRNA expression profiles of RCC 
and tumor-adjacent non-tumor tissues were assessed by lncRNA microarrays. The up-regulated lncRNAs were validated for their expression levels in another cohort of patients. In addition, we characterized the pathologic relevance of the lncRNA TCONS_00006756, also termed HEIRCC (high-expressed in renal cell carcinoma), in RCC development and progression. Furthermore, mechanistic investigations into the function of HEIRCC in RCC were performed through loss-of-function studies, which provided new insights into the role of lncRNAs in RCC development and progression. The current findings suggest a potential application for HEIRCC in the treatment of RCC.

A

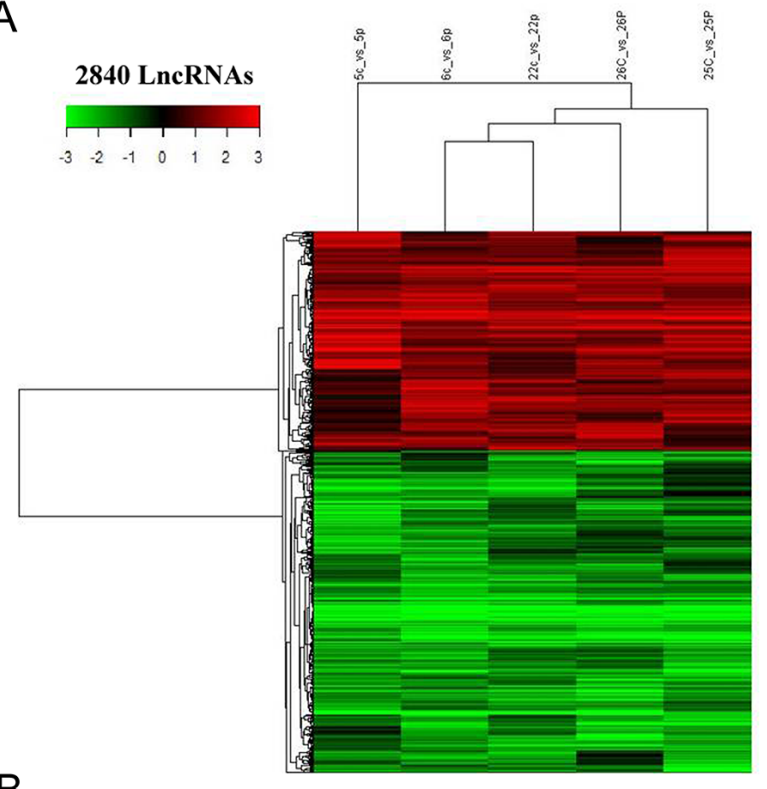

B

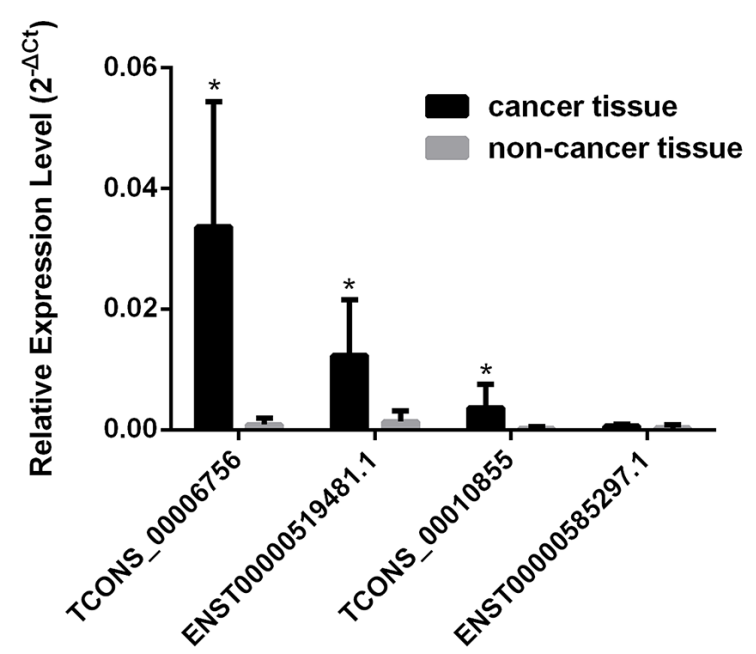

\section{RESULTS}

\section{HEIRCC is strongly up-regulated in the RCC tumor tissues and cancer cells}

Hierarchical clustering showed 2,840 (10\%) IncRNAs that were differentially expressed (cutoff fold change value, $2 ; P<0.05$ ) between cancer samples and non-tumor specimens. These comprised 1,151 upregulated and 1,688 down-regulated. Further, a total of $2,056(7.4 \%)$ protein-coding mRNAs were consistently increased; meanwhile, 1,961 (7.05\%) mRNAs showed decreased amounts (Figure 1A). These microarray
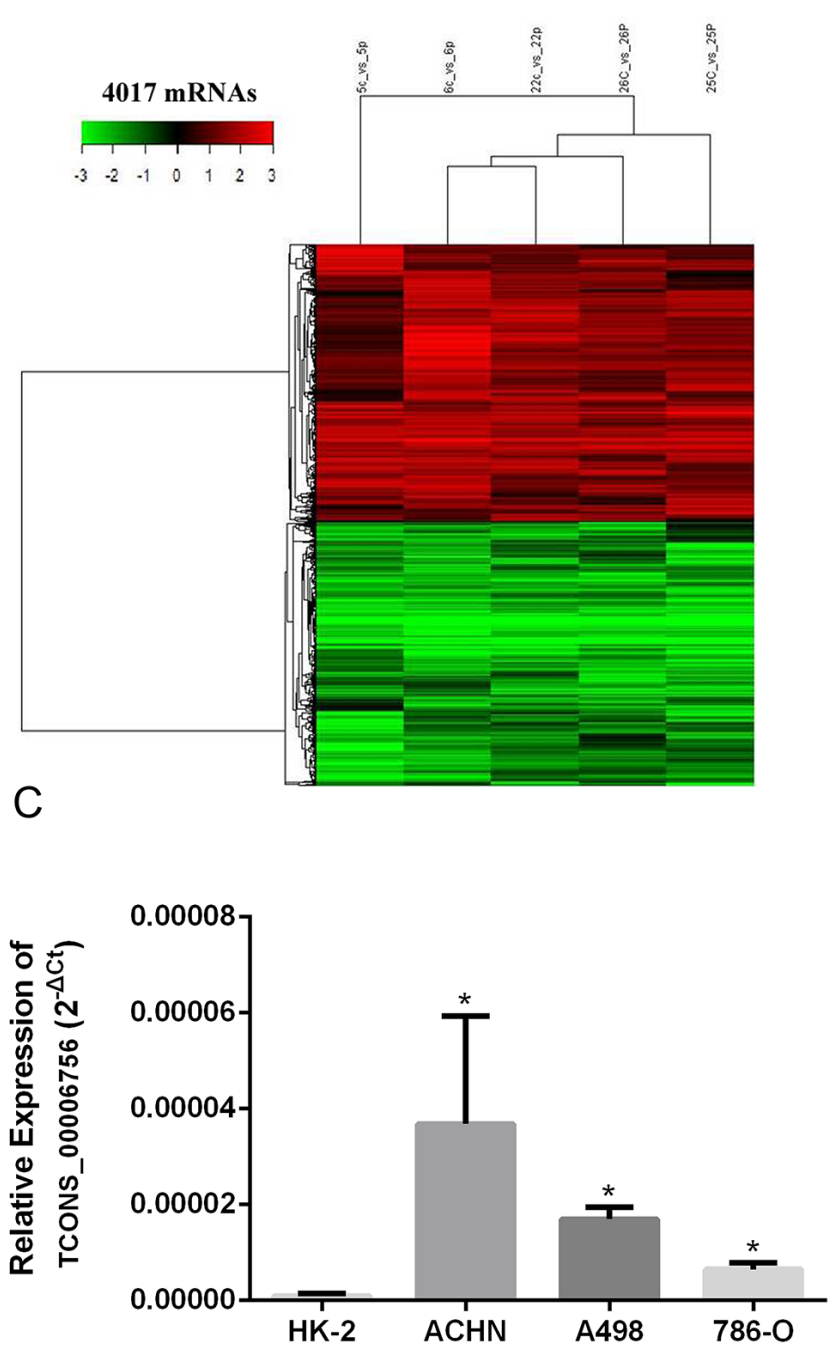

Figure 1: HEIRCC expression in RCC cell lines and tissues. A. Hierarchical clustering analysis of lncRNAs and mRNAs that were differentially expressed ( $>2$-fold; $P<0.05$ ) in 5 pairs of RCC and nontumor samples (c, cancer tissues; p, paired nontumor samples; 5-6-22-25-26, patient number). Red represents high expression and green represents low expression. B. The expression of four selected lncRNAs including HEIRCC in RCC patients. C. The relative expression of HEIRCC mRNA in renal cancer and normal cell line. ${ }^{*} P<0.05$. 
Table 1: Correlation between HEIRCC expression and clinicopathologic factors in RCC patients $(n=60)$

\begin{tabular}{|c|c|c|c|c|c|}
\hline \multirow{2}{*}{$\begin{array}{l}\text { Clinicopathological } \\
\text { parameters }\end{array}$} & \multirow[t]{2}{*}{ Case no. } & \multicolumn{2}{|c|}{ HEIRCC expression } & \multirow[t]{2}{*}{$X^{2}$} & \multirow[t]{2}{*}{$P$} \\
\hline & & High no. $(\%)$ & Low no.(\%) & & \\
\hline Age(years) & & & & 1.609 & 0.205 \\
\hline$\leq 60$ & 34 & $14(41.2 \%)$ & $20(58.8 \%)$ & & \\
\hline$>60$ & 26 & $15(57.7 \%)$ & $11(42.3 \%)$ & & \\
\hline Gender & & & & 0.601 & 0.438 \\
\hline Male & 29 & $13(44.8 \%)$ & $16(55.2 \%)$ & & \\
\hline Female & 31 & $17(54.8 \%)$ & $14(45.2 \%)$ & & \\
\hline Histological grade & & & & 4.356 & 0.037 \\
\hline Well & 45 & $19(42.2 \%)$ & $26(57.8 \%)$ & & \\
\hline Moderate \& Poor & 15 & $11(73.3 \%)$ & $4(26.7 \%)$ & & \\
\hline Tumor stage & & & & 3.937 & 0.047 \\
\hline I \& II & 47 & $18(38.3 \%)$ & $29(61.7 \%)$ & & \\
\hline III \& IV & 13 & $9(69.2 \%)$ & $4(30.8 \%)$ & & \\
\hline Lymph node metastasis & & & & 4.375 & 0.036 \\
\hline Positive & 10 & $7(70.0 \%)$ & $3(30.0 \%)$ & & \\
\hline Negative & 50 & $21(35.0 \%)$ & $39(65.0 \%)$ & & \\
\hline Distant metastasis & & & & 4.271 & 0.039 \\
\hline Positive & 12 & $9(75.0 \%)$ & $3(25.0 \%)$ & & \\
\hline Negative & 62 & $20(41.7 \%)$ & $28(58.3 \%)$ & & \\
\hline
\end{tabular}

results are available in NCBI Gene Expression Omnibus (accession number: GSE88811). The 100 lncRNAs and protein-coding genes showing the highest fold changes are summarized in Supplementary Table 1. Criteria with higher stringency were used to obtain lncRNAs for subsequent assessments: (1) elevated or reduced amounts; and (2) similar expression patterns in various specimens; (3) a fold-change in expression of $>10$. Interestingly, 4 and 7 lncRNAs were markedly downregulated and upregulated in RCC tissues, respectively. We focused mainly on the upregulated lncRNAs because they can be used more readily than down-regulated lncRNAs as early diagnosis markers or therapeutic targets. Some of them were further validated in an additional cohort of 55 samples by real-time PCR $(* P<0.05$; Figure $1 \mathrm{~B})$. A nonannotated transcript, TCONS_00006756, was identified as one of the most significantly up-regulated entities in RCC tumor tissues in comparison with corresponding adjacent tissue specimens. As shown in Figure 1C, the expression levels of TCONS_00006756 were starkly elevated in RCC cells compared with HK-2 amounts $(* P<0.05)$. These findings suggested that TCONS_00006756 might act as an oncogenic lncRNA in RCC carcinogenesis; the IncRNA is hereafter referred to as HEIRCC. Information from the
GenBank revealed that HEIRCC is located on the reverse strand of human chromosome 3:194021490-194023848, and has a $340 \mathrm{bp}$ long transcript.

\section{HEIRCC expression is correlated with RCC progression}

To determine whether HEIRCC expression levels are related to RCC progression, we analyzed the association of HEIRCC with clinicopathological factors in RCC patients. Statistical analyses revealed that high expression of HEIRCC in RCC patients was significantly correlated with tumor stage, histological grade, lymph node metastasis, and distant metastasis, as shown in Table $1(P<0.05)$. Therefore, overexpression of HEIRCC is involved in RCC malignancy.

\section{HEIRCC affects cell proliferation and apoptosis}

To further assess the biological effect of HEIRCC in RCC malignancy, loss-of-function studies were performed. HEIRCC expression levels were efficiently reduced in cancer cells transfected with HEIRCC-siRNA\#2. Therefore, HEIRCC-siRNA\#2 was used in subsequent functional 
experiments ( ${ }^{*} P<0.05$, Figure $\left.2 \mathrm{~A}, 2 \mathrm{~B}\right)$. Interestingly, in vitro CCK-8 assays demonstrated that HEIRCC knockdown dramatically reduced the proliferative capacity of RCC cells in comparison with the siRNA scramble control $(* P$ $<0.05$, Figure 2C, 2D). Next, we analyzed cell apoptosis by flow cytometry. Increased apoptosis was observed in RCC cells transfected with HEIRCC-siRNA\#2 ( $* P<0.05$, Figure 2E, 2F). Collectively, these findings suggested that HEIRCC may play a pivotal role in the proliferation and apoptosis of RCC cells in vitro.
A

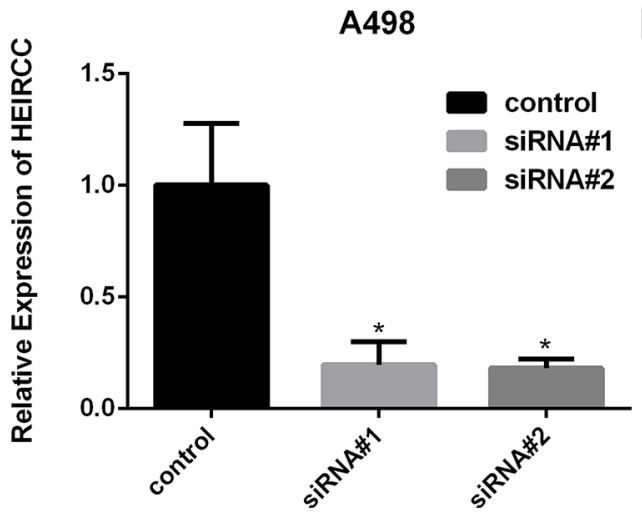

C

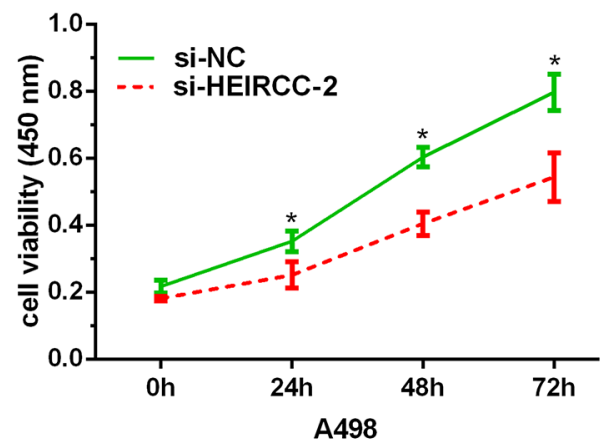

$\mathbf{E}$

A498 si-NC

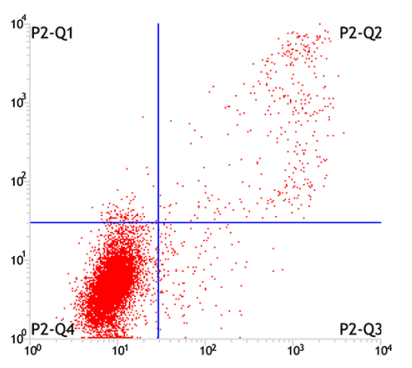

ACHN si-NC

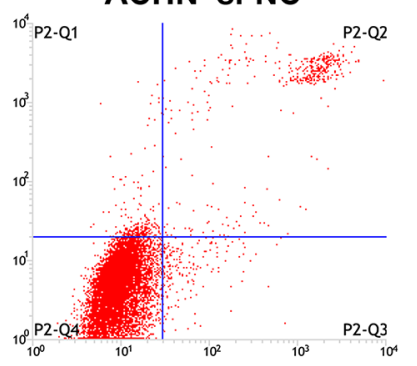

A498 si-HEIRCC-2
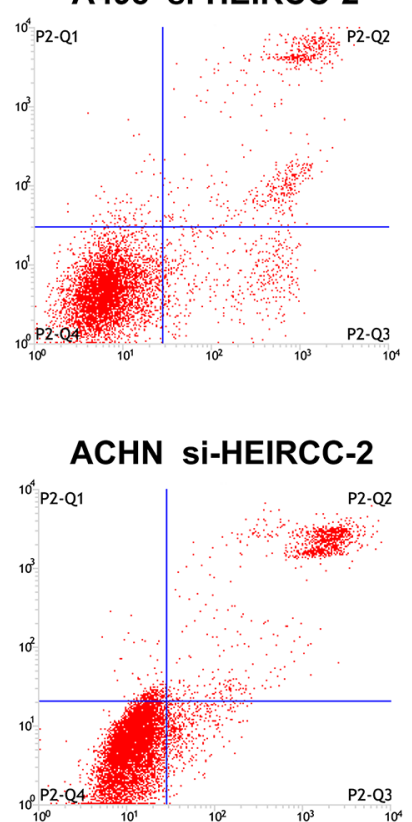

B
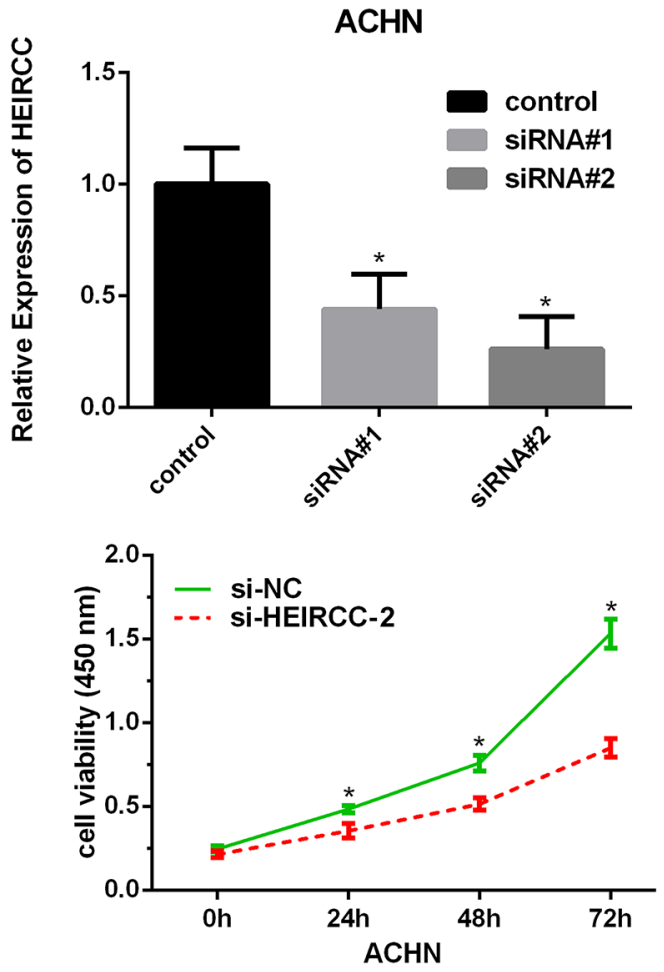

$\mathbf{F}$

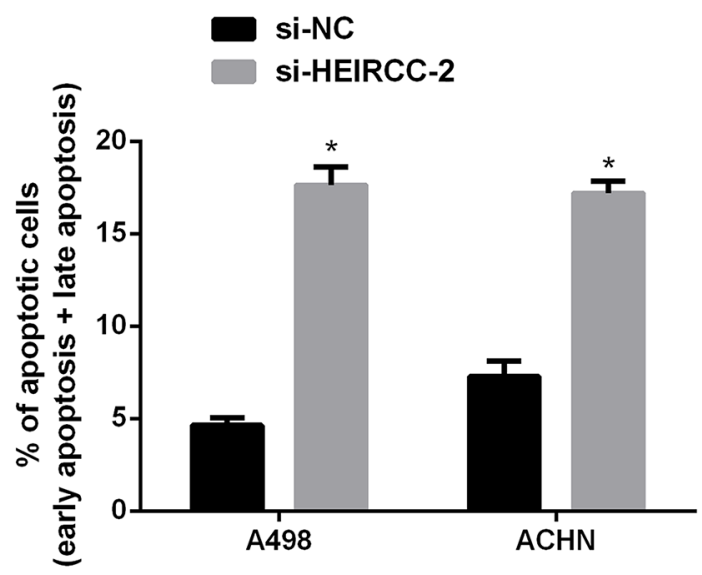

Figure 2: Effects of HEIRCC on RCC cell proliferation and apoptosis. A, B. Decreased expression of HEIRCC was detected after transfection of HEIRCC-siRNA and siRNA control. C, D. HEIRCC depletion inhibited the proliferation of A498 and ACHN. E, F. HEIRCC knockdown promoted RCC cells apoptosis. ${ }^{*} P<0.05$. 


\section{HEIRCC is involved in RCC cell migration and invasion}

Cell migration and invasion are important prerequisites for tumor progression and metastasis. To assess whether HEIRCC modulates cell migration and invasion, wound healing test and matrigel invasion assay were performed. Our results demonstrated that RCC cell malignancy features were significantly suppressed after HEIRCC silencing by HEIRCC-siRNA\#2 $\left({ }^{*} P<0.05\right.$, Figure 3A, 3B, 3C).

\section{HEIRCC regulates RCC cell progression via the EMT mechanism}

Since EMT is crucial for tumor propagation and metastasis, the effects of HEIRCC on EMT were analyzed. Interestingly, depletion of HEIRCC resulted in upregulated E-cadherin as well as downregulated
$\mathrm{N}$-cadherin and Vimentin. In addition, EMT transcription factors were analyzed, and partial loss of endogenous HEIRCC expression overtly decreased ZEB1 amounts, whereas Snail and Slug amounts showed no remarkable changes. Overall, the above findings pointed to HEIRCC as a new positive regulator of EMT, with a function in RCC malignancy (Figure 4).

\section{DISCUSSION}

Despite the great therapeutic advances made in RCC, including surgical resection and adjuvant therapy, the longterm prognosis of RCC patients with distant metastases remains unfavorable. Increasing functional studies have indicated that lncRNAs, which may act as oncogenes or tumor suppressors, are involved in the carcinogenesis and progression of several tumors, including RCC [11, 12]. Although dysregulation of lncRNAs associated with RCC has been demonstrated, the functions and clinical significance
A

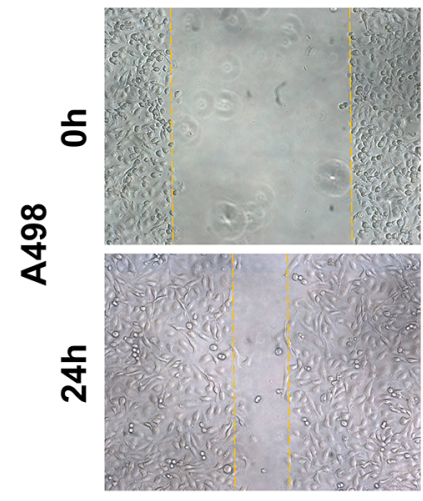

B

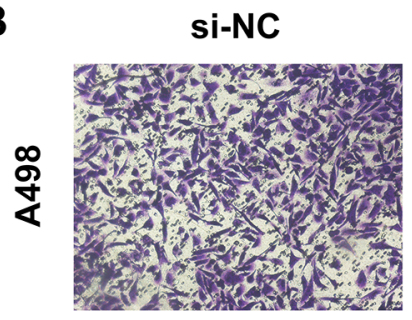

si-HEIRCC-2

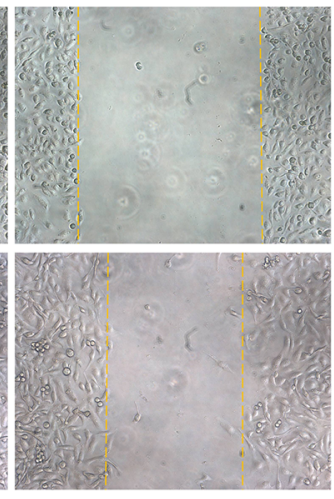

si-HEIRCC-2

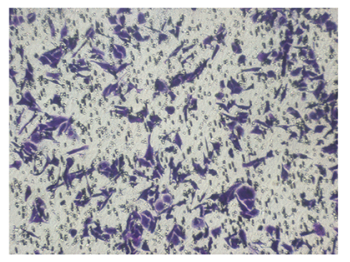

A498

C

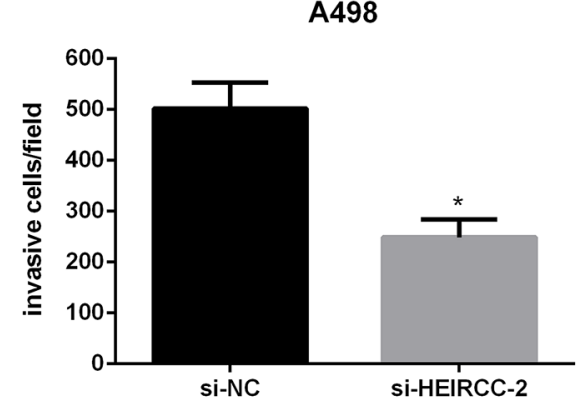

si-NC

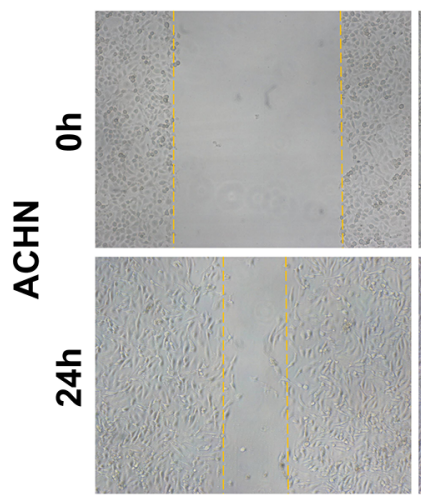

si-NC

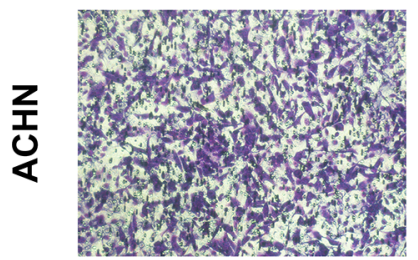

si-HEIRCC-2

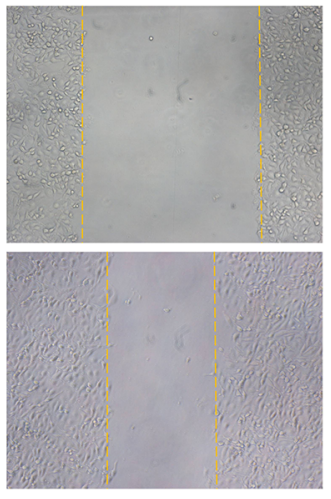

si-HEIRCC-2

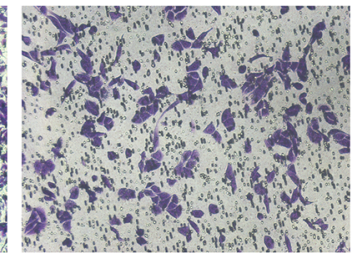

ACHN

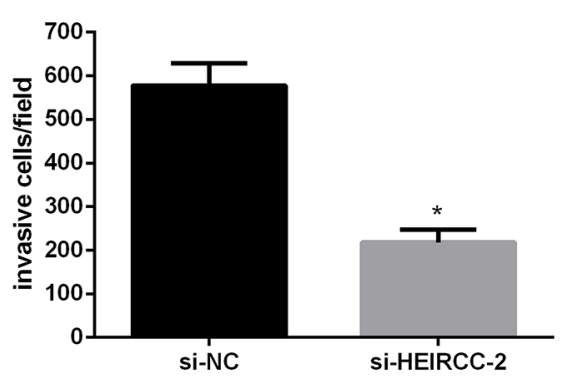

Figure 3: Effects of HEIRCC on RCC cell progression. A. The migration and B,C invasion capacity of A498 and ACHN cells was decreased by HEIRCC-siRNA. $P<0.05$. 
of most dysregulated IncRNAs in the progression and aggressiveness of RCC remain unknown $[13,14]$.

In the current study, we identified the novel oncogenic lncRNA TCONS_00006756, termed HEIRCC, as one of the most up-regulated lncRNAs in RCC tissues compared with paired peritumoral tissues by lncRNA microarray analysis. This is consistent with previous studies by Blondeau et al. and $\mathrm{Yu}$ et al. $[15,16]$. Moreover, further validation was performed in an additional cohort of RCC clinical tissue specimens and cell lines. High expression of HEIRCC is closely associated with large tumors, poor differentiation, and lymph node metastasis, and potentially correlated with RCC formation and progression.

Moreover, loss-of-function studies suggested HEIRCC silencing results in reduced cell growth, enhanced apoptosis, and inhibited aggressiveness. The above findings indicated HEIRCC might be a tumor oncogene in RCC.
EMT is crucial in normal organ development and human pathology, e.g. tumor aggressiveness. It is generally accepted that EMT is critical for tumor invasion as well as metastasis [17, 18, 19]. However, the underlying molecular mechanisms of EMT mediating the metastatic cascade remain largely unclear. Accumulating evidence indicates that lncRNAs can drive metastasis by inducing migration and invasion of cell through regulation of critical proteins [20]. In this study, we surprisingly observed that after HEIRCC knockdown, some hallmarks of mesenchymal cells such as N-cadherin and Vimentin showed reduced levels whereas E-cadherin (epithelial protein) amounts were increased. It has been reported that several transcription factors, including Snail, Slug and ZEB1 can initiate EMT via repression of E-cadherin expression by targeting the E-boxes in its promoter [21, 22]. We thus assessed whether HEIRCC regulates these transcription factors, and promotes tumor progression and metastasis in RCC. Western

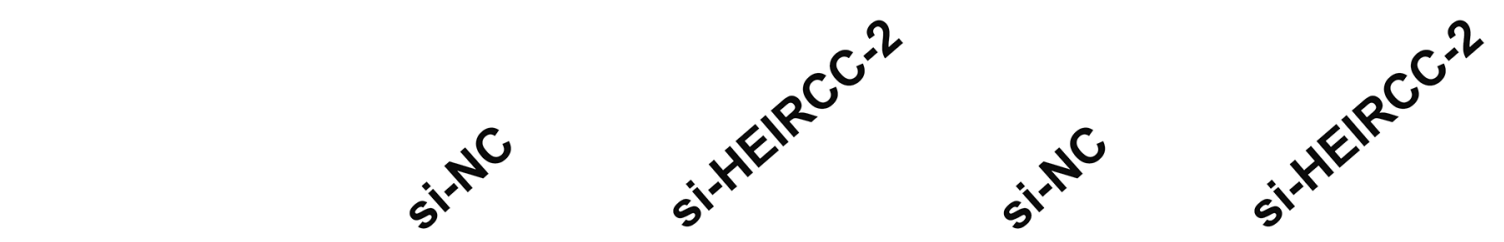

\section{E-Cadherin}

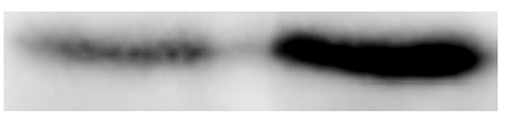

N-Cadherin

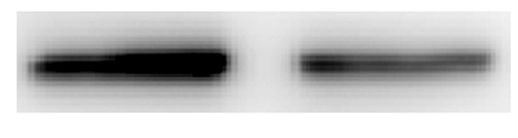

Vimentin

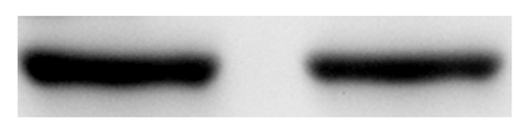

ZEB-1

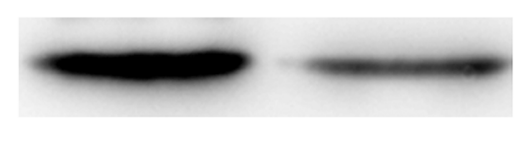

Snail

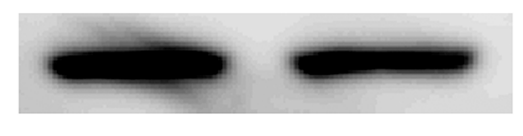

Slug

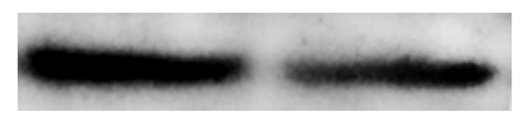

$\beta$-actin

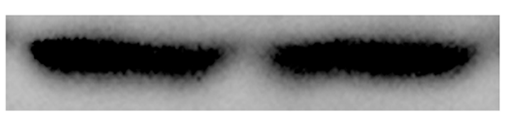

A498

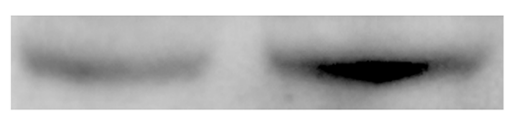

135KDa

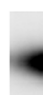

140KDa

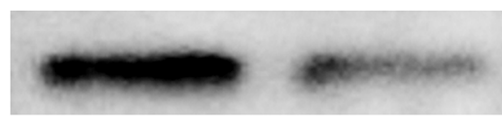

54KDa

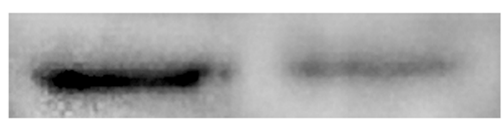

124KDa

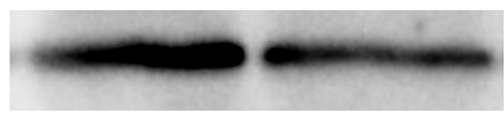

29KDa

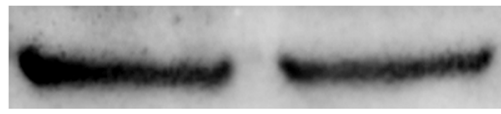

35KDa

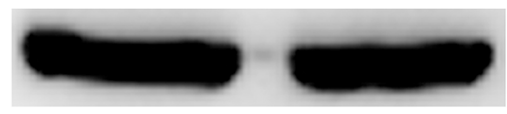

\section{KDa}

Figure 4: The expression of EMT related proteins were measured by western blot analyses after transfection. 
blotting analysis showed that knockdown of HEIRCC markedly repressed ZEB1 expression levels, while the other transcription factors such as Snail and Slug were not significantly changed. Overall, these findings indicated that HEIRCC promotes RCC progression by activating the EMT process in a ZEB1-dependent manner.

In summary, we have described, for the first time, the clinicopathologic features as well as the critical function of HEIRCC in RCC cell migration, invasion, and EMT. Our findings suggest that HEIRCC may serve as a novel therapeutic target for the advanced RCC treatment. Owing to the limited information obtained with a single siRNA, more specific siRNAs with high inhibition efficiency and a gain-of-function HEIRCC model system will be applied to comprehensively evaluate its biological function in subsequent studies. Moreover, the correlation between HEIRCC and the 5-year survival rate needs to be established with more patients.

\section{MATERIALS AND METHODS}

\section{Patients and specimens}

5 paired samples were prepared for lncRNA microarray analysis and 60 paired samples were used for an extra evaluation by real-time PCR. All patients were informed and have declared written informed consent that their samples can be used for research. This study was approved by the Research Ethics Committee of Chongqing Medical University, China. Patients received retroperitoneal laparoscopic radical nephrectomy, retroperitoneal laparoscopic partial nephrectomy, cytoreductiv enephrectomy at The First Affiliated Hospital of Chongqing Medical University and were diagnosed with ccRCC histopathologically after surgery. The tumor specimens were staged primarily according to UICC 2009 TNM tumor staging system [23], and histological graded based on the Fuhrman criteria and Union Internationale Contre le Cancer [24, 25]. All the samples were rapidly and carefully frozen in liquid nitrogen after resection until use. None of the patients had been pretreated with radiotherapy or chemotherapy prior to surgery.

\section{Cell culture}

786-O, A498, ACHN RCC cell lines and immortalized normal human proximal tubule epithelial cell lines HK-2 were obtained from the Cell Bank of Type Culture Collection (Chinese Academy of Sciences, Shanghai, China). Cells were maintained at $37^{\circ} \mathrm{C}$ in a humidified incubator containing 5\% $\mathrm{CO}_{2}$ in RPMI 1640 medium supplemented with $10 \%$ fetal bovine serum and antibiotics.

\section{Microarray and computational analysis}

The microarray work was performed by CapitalBio Corporation, Beijing, China. Briefly, paired samples were used to synthesize the double-stranded complementary DNA (cDNA) by reverse-transcription polymerase chain reaction. The labeled cDNAs were hybridized to the Agilent Human LncRNA + messenger RNA (mRNA) Array V4.0 ( 4 x 180K, format). More than 40,916 IncRNAs were collected from authoritative data sources, including the GENCODE/Ensembl, Human LncRNA Catalog, RefSeq, USCS, ncRNA Expression Database (NRED), H-InvDB, LncRNAs-a (enhancer-like), RNAdb, CombinedLit, Antisense ncRNA pipeline, HoxncRNAs, UCRs, and Chen Runsheng lab. After hybridization and washing, the processed slides were scanned with the Agilent Microarray Scanner (Agilent technologies Santa Clara, CA, USA). Feature Extraction software 11.0.1.1 (Agilent Technologies) was used to analyze array images. Raw data were normalized by a Quantile algorithm using GeneSpring GX v11.5.1 software package (Agilent Technologies). A fold change of $\geq 2.0$ and $\mathrm{P} \leq 0.05$ was set as the threshold for statistically significant up- and down-regulated genes, respectively. The FDR were calculated using Benjamini Hochberg method throughout the computational analysis. Hierarchical Clustering was performed based on differentially-expressed mRNAs and IncRNAs using Cluster_Treeview software from Stanford University.

\section{Real-time quantitative reverse transcription- PCR}

Total RNA from 786-O, A498, ACHN and HK-2, as well as the RCC samples, and corresponding adjacent noncancerous kidney samples was isolated by RNAiso plus (TaKaRa, Dalian, China). Then RNA was reversely transcribed using a PrimeScript ${ }^{\mathrm{TM}}$ RT reagent Kits with gDNA Eraser (TaKaRa, Dalian, China). Real-time PCR was performed using the SYBR Green PCR Kit purchased from TaKaRa Biotechnology. The primers were designed and synthesized by Genepharma Co., Ltd. (Shanghai, China). GAPDH (forward 5'-GTCATCAATGGAAATCCCATCA-3'; reverse 5'-CCAG TGGACTCCACGACGTAC-3' Size: 98bp); ENST 00000519481.1 (forward 5'-TCTGAGCCTGATGGA TTTACAGTGA-3'; reverse 5'-TGTCATTCCAGTG CATGGTTCC-3' Size:76bp); TCONS_00006756 (forward 5'-GCTGCTATTCTGGTGCCC-3'; reverse 5'-TCAACT CCGATAAACAGGTGA-3' Size:159bp); TCONS_0001 0855 (forward 5'-AGAGGGTAGGGGATGGAAGTGG-3'; reverse 5'-AGGGATAGCAAACACCTAAAATGCA-3' Size:143bp); ENST00000585297.1 (forward 5'-AGTTGGAA GGACTCCCCAGG-3'; reverse 5'-TAACCCACAAATG AAGTGCTACTCT-3' Size:143bp).

\section{RNA interference and cell transfection}

Specific siRNAs targeting TCONS_00006756 and a scrambled negative control (si-NC) were synthesized by Genepharma Co., Ltd. (Shanghai, China). A498 and ACHN 
were seeded onto the plate and reached $40 \%$ confluence on the second day, the siRNAs were transfected into the cells using Lipofectamine 2000 reagents (Life Technologies) according to the manufactuer's instruction. The efficiencies of RNA interference were determined by qRTPCR after 48h transfection. TCONS_00006756-siRNA\#1: GGAAGACUGUCACCCUCUUTT; TCONS_00006756SiRNA\#2: UCACCUGUUUAUCGGAGUUTT.

\section{Cell proliferation assay}

Appropriate cells that had been transfected with TCONS_00006756 siRNAs and negative control (NC) were seeded into 96-well plates in triplicate at densities of approximately 2000 cells per well. CCK-8 (Wanleibio, China) assay was used to analyze the cell viability at the indicated times.

\section{Flow cytometry analysis for apoptosis}

After $48 \mathrm{~h}$ transfection as earlier described, the cells were collected and washed as well as resuspended with PBS. Then, cells were stained with FITC-Annexin V and PI (Beyotime Biotechnology, China) for apoptosis analysis with a FACScan flow cytometer (BD Bioscience). The experiments were performed in triplicates.

\section{Wound scratch assay}

Cells from each group were seeded into 24-well plates at a density that reached $90-95 \%$ confluence as a monolayer. The monolayer was gently scratched across the center of the well with a $200 \mu \mathrm{l}$ plastic tip. After scratching, images were obtained at 0 and $24 \mathrm{~h}$ by using a microscope.

\section{Transwell invasion assay}

Matrigel (BD Biosciences, USA) was thawed at $-4^{\circ} \mathrm{C}$ overnight and diluted with serum-free RPMI-1640 medium (dilution, 1:8). A $60-\mu 1$ sample of this mixture was deposited evenly into a 24-well chamber $(8 \mu \mathrm{m}$, Millipore, USA), which was incubated for $30 \mathrm{~min}$ at $37^{\circ} \mathrm{C}$. A total of $5 \times 10^{4}$ cells were resuspended in $200 \mu \mathrm{l}$ of serum-free culture medium and loaded into the upper chamber (Millipore) whereas the bottom chamber was filled with $500 \mu \mathrm{l}$ culture medium containing 10\% FBS. The setup was incubated in the $37^{\circ} \mathrm{C}$ humidified $\mathrm{CO}_{2}$ for $48 \mathrm{~h}$. Cells that passed through the filter were fixed with $4 \%$ paraformaldehyde and stained with $0.5 \%$ crystal violet. The numbers of invaded cells were counted in five randomly selected high power fields under a microscope (Olympus).

\section{Western blot and antibodies}

Total proteins were lysed in RIPA buffer (Beyotime Biotechnology) supplemented with protease inhibitors.
The protein concentrations were determined using BCA Protein Assay Kit (Beyotime Biotechnology). Identical quantities of proteins were separated on $10 \%$ SDS-PAGE and transferred to a PVDF membrane. After incubation with antibodies specific for Vimentin, N-Cadherin, and E-Cadherin, Slug, ZEB1, Snail and $\beta$-actin (Wanleibio, China). The bands were then scanned by a Imaging System and quantified by Quantity One v4.6.2 software (BIO-RAD).

\section{Statistical analysis}

All the statistical analyses were performed using SPSS 19.0 software (SPSS, Chicago, IL, USA). All data were summarized and expressed as the mean \pm SD. The differences between independent groups were analyzed using Student's $t$-test. Pearson's chi-square tests were used to analyse the correlation between lncRNA levels and clinical features. A $P$ value less than 0.05 was considered to be statistically significant.

\section{ACKNOWLEDGMENTS AND FUNDING}

The authors would like to thank Ying Liu, Department of Preventive Medicine, Hubei University of Medicine, for her statistical analysis. This work was supported by the Chongqing Science and Technology Program Foundation(No. 2013yykfA110004).

\section{CONFLICTS OF INTEREST}

The authors declare no competing financial interests.

\section{REFERENCES}

1. Rini BI, Rathmell WK, Godley P. Renal cell carcinoma. Curr Opin Oncol. 2008. 20: 300-6.

2. Pantuck AJ, Zisman A, Belldegrun AS. The changing natural history of renal cell carcinoma. J Urol. 2001. 166: 1611-23.

3. Motzer RJ, Russo P. Systemic therapy for renal cell carcinoma. J Urol. 2000. 163: 408-17.

4. Greenburg G, Hay ED. Epithelia suspended in collagen gels can lose polarity and express characteristics of migrating mesenchymal cells. J Cell Biol. 1982. 95: 333-9.

5. Acloque H, Thiery JP, Nieto MA. The physiology and pathology of the EMT. Meeting on the epithelialmesenchymal transition. EMBO Rep. 2008. 9:322-6.

6. Guttman M, Rinn JL. Modular regulatory principles of large non-coding RNAs. Nature. 2012. 482: 339-46.

7. Gutschner T, Diederichs S. The hallmarks of cancer: a long non-coding RNA point of view. RNA Biol. 2012. 9: 703-19.

8. Prensner JR, Chinnaiyan AM. The emergence of lncRNAs in cancer biology. Cancer Discov. 2011. 1: 391-407. 
9. Zhang X, Gejman R, Mahta A, Zhong Y, Rice KA, Zhou Y, Cheunsuchon P, Louis DN, Klibanski A. Maternally expressed gene 3, an imprinted noncoding RNA gene, is associated with meningioma pathogenesis and progression. Cancer Res. 2010. 70: 2350-8.

10. Takahashi Y, Sawada G, Kurashige J, Uchi R, Matsumura T, Ueo H, Takano Y, Eguchi H, Sudo T, Sugimachi K, Yamamoto H, Doki Y, Mori M, Mimori K. Amplification of PVT-1 is involved in poor prognosis via apoptosis inhibition in colorectal cancers. Br J Cancer. 2014. 110: 164-71.

11. Sultmann H, Diederichs S. Long noncoding RNA: "LNCs" to cancer. Eur Urol. 2014. 65: 1152-3.

12. Svoboda M, Slyskova J, Schneiderova M, Makovicky P, Bielik L, Levy M, Lipska L, Hemmelova B, Kala Z, Protivankova M, Vycital O, Liska V, Schwarzova L, et al. HOTAIR long non-coding RNA is a negative prognostic factor not only in primary tumors, but also in the blood of colorectal cancer patients. Carcinogenesis. 2014. 35: 1510-5.

13. Martens-Uzunova ES, Bottcher R, Croce CM, Jenster G, Visakorpi T, Calin GA. Long noncoding RNA in prostate, bladder, and kidney cancer. Eur Urol. 2014. 65: 1140-51.

14. Tsai MC, Manor O, Wan Y, Mosammaparast N, Wang JK, Lan F, Shi Y, Segal E, Chang HY. Long noncoding RNA as modular scaffold of histone modification complexes. Science. 2010. 329: 689-93.

15. Blondeau JJ, Deng M, Syring I, Schrödter S, Schmidt D, Perner S, Müller SC, Ellinger J. Identification of novel long non-coding RNAs in clear cell renal cell carcinoma. Clin Epigenetics. 2015. 7: 10.

16. Yu G, Yao W, Wang J, Ma X, Xiao W, Li H, Xia D, Yang Y, Deng K, Xiao H, Wang B, Guo X, Guan W, et al. LncRNAs expression signatures of renal clear cell carcinoma revealed by microarray. PLoS One. 2012. 7: e42377.
17. Greenburg G, Hay ED. Epithelia suspended in collagen gels can lose polarity and express characteristics of migrating mesenchymal cells. J Cell Biol. 1982. 95: 333-9.

18. Acloque H, Thiery JP, Nieto MA. The physiology and pathology of the EMT. Meeting on the epithelialmesenchymal transition. EMBO Rep. 2008. 9: 322-6.

19. Thiery JP. Epithelial-mesenchymal transitions in tumour progression. Nat Rev Cancer. 2002. 2: 442-54.

20. Dhamija S, Diederichs S. From junk to master regulators of invasion: lncRNA functions in migration, EMT and metastasis. Int J Cancer. 2016. 139:269-80.

21. Otsuki S, Inokuchi M, Enjoji M, Ishikawa T, Takagi Y, Kato K, Yamada H, Kojima K, Sugihara K. Vimentin expression is associated with decreased survival in gastric cancer. Oncol Rep. 2011. 25:1235-1242.

22. Rosivatz E, Becker I, Specht K, Fricke E, Luber B, Busch R, Höfler H, Becker KF. Differential expression of the epithelial-mesenchymal transition regulators snail, SIP1, and twist in gastric cancer. Am J Pathol. 2002. 161:1881-1891.

23. Kim SP, Alt AL, Weight CJ, Costello BA, Cheville JC, Lohse C, Allmer C, Leibovich BC. Independent validation of the 2010 American Joint Committee on Cancer TNM classification for renal cell carcinoma: results from a large, single institution cohort. J Urol. 2011. 185:2035-2039.

24. Störkel S, Eble JN, Adlakha K, Amin M, Blute ML, Bostwick DG, Darson M, Delahunt B, Iczkowski K. Classification of renal cell carcinoma: Workgroup No. 1. Union Internationale Contre le Cancer (UICC) and the American Joint Committee on Cancer (AJCC). Cancer. 1997. 80:987-9.

25. Eble JN, Sauter G, Epstein JI. (2004) World Health Organization classification of tumors. Pathology and genetics of tumors of the urinary system and male genital organs. IARC Press, Lyon, pp 16-18. 